\title{
SCATTERED SPACES, COMPACTIFICATIONS AND AN APPLICATION TO IMAGE CLASSIFICATION PROBLEM
}

\author{
Monerah Al-Hajri — Karim Belaid — Lamia JaAfar Belaid
}

\begin{abstract}
In this paper, we characterize spaces such that their one-point compactification is a scattered space. We study spaces such that their Wallman compactification is scattered and we give necessary and sufficient conditions on the subspace $A$ to get its closure scattered. An application of scattered spaces to image classification problem is also presented.
\end{abstract}

\section{Introduction}

A topological space $X$ is said to be scattered if every non-empty subset $S$ of $X$ contains at least one point which is isolated in $S$ (that is, $X$ is scattered if and only if it contains no non-empty subset which is dense-in-itself).

Compact scattered spaces have found important use in analysis and topology (see for example [10] in which S. M r ow k a, M. R a j a g o p a l a n, T. S o u n d a rarajan characterized compact scattered Hausdorff spaces). On the other hand, from 2004 to 2015 K. B e l a i d et al. [1, 4], 5] studied some topological spaces and compactifications.

Section 2 is devoted to a short study of digital spaces and to a characterization of the subspace $A$ to get its closure scattered.

Section 3 deals with the problem when the one-point compactification (resp. Wallman compactification) is a scattered space.

The purpose of Section 4 is to start a study about spaces such that their one-point compactification is a $S P$-scattered space. Moreover, we consider some numerical applications of scattered spaces to image classification problem in this section.

(C) 2016 Mathematical Institute, Slovak Academy of Sciences. 2010 Mathematics Subject Classification: Primary 06B30, 06F30; Secondary 54F05. Keywords: classification, compactification, digital topology, scattered spaces.

Supported by Deanship of Scientific research of the University of Dammam under the reference 2015185. 


\section{Scattered subspaces and digital topology}

First, recall the definition of the digital line: The digital line (called also Khalimsky line) is the set of integers $\mathbb{Z}$ equipped by the topology $\mathcal{K}$, generated by $\mathcal{G}=\{\{2 n-1,2 n, 2 n+1\} \mid n \in \mathbb{Z}\}[\mathbb{8}$.

Proposition 2.1. The digital line is a scattered space.

Pro of. Let $S$ be a subset of $\mathbb{Z}$. If there exists an odd integer $k \in S$, then $\{k\}$ is an open set of $\mathbb{Z}$; so that $k$ is an isolated point in $S$. Suppose now, all elements of $S$ are even. Hence for $k \in S,\{k\}$ is an open set of $S$. Thus $k$ is an isolated point in $S$. Therefore the digital line is a scattered space.

Now, we recall the definition of the digital plane (also called the Khalimsky plane).

Definition 2.2. The Khalimsky topology on the set $\mathbb{Z}^{2}$ is the topology $\mathcal{K}$ generated by: For any $z=(x, y) \in \mathbb{Z}^{2}$

- if $x, y$ are even, $\{(x-i, y+k) \mid i, k \in\{-1,0,1\}\}$,

- if $x$ is even and $y$ is odd $\{(x+k, y) \mid k \in\{-1,1\}\}$,

- if $x$ is odd and $y$ is even $\{(x, y+k) \mid k \in\{-1,1\}\}$,

- $\{z\}$ otherwise.

The set $\mathbb{Z}^{2}$ equipped with the Khalimsky topology is called the digital plane.

Proposition 2.3. The digital plane is a scattered space.

Proof. Let $S$ be a subset of $\mathbb{Z}^{2}$. Suppose that there exists $z \in S$ such that $z=(x, y)$ where $x$ and $y$ are odd. Then $\{z\}$ is an open set of $\mathbb{Z}^{2}$; so that $z$ is an isolated point in $S$. Suppose that $S$ does not contain such an element and suppose that there exists $z \in S$ such that $z=(x, y)$ with either $x$ odd and $y$ even or $x$ even and $y$ odd. Hence $\{z\}$ is an open set of $S$; so that $z$ is an isolated point in $S$. Finally, if every element $z=(x, y)$ of $S$ with $x, y$ is even, then $\{z\}$ is an open set of $S$; so that $z$ is an isolated point in $S$.

Definition 2.4. Let $X$ be a topological space. A subset $S$ of $X$ is called a scattered subset of $X$ if $S$ considered as a topological space is scattered.

The following result is an immediate consequence of the fact that an isolated point in the closure of a subset $N$ of a topological space $X$ is an element of $N$; so that it is an isolated point of $N$.

Proposition 2.5. Let $X$ be a topological space and $N \subseteq X$. If $\bar{N}$ is scattered, then $N$ is scattered. 


\section{SCATTERED SPACES}

EXAMPLE 2.6. The closure of a scattered subset is not necessary scattered. Let $\left(X_{1}, T_{1}\right)$ be the digital line and $X_{2}$ be the set of integers. Let $Y$ be the disjoint union of $X_{1}$ and $X_{2}$ equip $Y$ with the following topology:

$$
T=\{Y\} \cup\left\{O \mid O \in T_{1}\right\} \cup\left\{X_{1} \cup(\downarrow x) \mid x \in X_{2}\right\},
$$

where $(\downarrow x)=\left\{y \in X_{2} \mid y \leq x\right\}$.

Let $N=X_{1}$. Then $N$ is a scattered set. It is immediate that $\bar{N}=Y$. Let $S=X_{2}$. Since $X_{2}$ has no isolated points, $\bar{N}$ is not scattered.

Proposition 2.7. Let $X$ be a $T_{0}$-space and $N$ be a subset of $X$. Then $\bar{N}$ is scattered if and only if $N$ and $\bar{N}-N$ are scattered.

P r o of. It is clear that if $\bar{N}$ is scattered then $N$ and $\bar{N}-N$ are scattered.

Suppose now that $N$ and $\bar{N}-N$ are scattered and let $S$ be a subset of $\bar{N}$. We consider two cases:

- Case 1: $S \subseteq N$ or $S \subseteq \bar{N}$. Then there exists an element $z$ of $S$ and an open set $O$ of $X$ such that $O \cap S=\{z\}$.

- Case 2: $S \cap N \neq \emptyset$ and $S \cap \bar{N} \neq \emptyset$. Since $N$ is scattered and $S \cap N \neq \emptyset$, there exists an element $z$ of $S \cap N$ and an open set $O$ of $X$ such that $O \cap(S \cap N)=\{z\}$. Suppose that $(O \cap S) \cap(\bar{N}-N) \neq \emptyset$. Since $\bar{N}-N$ is scattered, there exists an element $x$ of $(O \cap S) \cap(\bar{N}-N)$ and an open set $U$ of $X$ such that $U \cap((O \cap S) \cap(\bar{N}-N))=\{x\}$. Hence either $S \cap U=\{x\}$ or $S \cap U=\{x, z\}$. It is immediate that if $S \cap U=\{x\}$ then $x$ is an isolated point of $S$. Suppose that $S \cap U=\{x, z\}$. Since $X$ is a $T_{0}$-space and $x \in \bar{N}-N$, there exists an open set $V$ of $X$ such that $z \in V$ and $x \notin V$. Hence $(V \cap O \cap U) \cap S=\{z\}$. Thus $S$ is scattered.

Proposition 2.8. Let $X$ be a $T_{0}$-space and $S_{1}, S_{2}$ be two scattered subspaces of $X$. Then the following statements hold:

(1) $S_{1} \cap S_{2}$ is a scattered subset of $X$.

(2) $S_{1} \cup S_{2}$ is a scattered subset of $X$.

Pro of. We have,

(1) Straightforward.

(2) Since $S_{1}$ is a scattered subspace of $X$, there exists $x \in S_{1}$ and an open neighborhood $O$ of $x$ such that $S_{1} \cap O=\{x\}$. If $O \cap S_{2}=\emptyset$, then $O \cap\left(S_{1} \cup S_{2}\right)=\{x\}$; so that $x$ is an isolated point of $S_{1} \cup S_{2}$.

Suppose that $O \cap S_{2} \neq \emptyset$. Since $O \cap S_{2}$ is a subset of the scattered subspace $S_{2}$ of $X$, there exists $y \in O \cap S_{2}$ and an open neighborhood $V$ of $y$ such that $\left(O \cap S_{2}\right) \cap V=\{y\}$. Hence $O \cap V=\{y\}$ or $O \cap V=\{x, y\}$. If $O \cap V=\{y\}$, then y is an isolated point of $S_{1} \cup S_{2}$. Since $X$ is a $T_{0}$-space, 
there exists an open set $W$ of $X$ such that $x \in W$ and $y \notin W$ or $y \in W$ and $x \notin W$. Hence $(O \cap V \cap W) \cap\left(S_{1} \cup S_{2}\right)=\{x\}$ or $(O \cap V \cap W) \cap\left(S_{1} \cup S_{2}\right)=\{x\}$. Thus $S_{1} \cup S_{2}$ has an isolated point. Therefore $S_{1} \cup S_{2}$ is a scattered subspace of $X$.

Corollary 2.9. Let $X$ be a topological space and $\left\{S_{i} \mid i \in I\right\}$ be a finite collection of scattered subspaces of $X$. Then $\cup_{i \in I} S_{i}$ is a scattered subspace of $X$.

EXAmPLE 2.10. The assumption that the collection is finite in the Corollary 2.9 is essential, in fact, the rational topological space $\mathbb{Q}$ is an infinite union of singletons; so that $\mathbb{Q}$ is an infinite union of scattered subspaces. It is immediate that $\mathbb{Q}$ is not a scattered space.

\section{Scattered spaces and compactifications}

First, recall that a compactification of a topological space $X$ is a compact topological space $K(X)$ such that the natural embedding $i: X \longrightarrow K(X)$ is continuous and the subspace $i(X)$ is a dense subspace of $K(X)$. Hence the topological space $X$ can be identified with $i(X)$. Thus $X$ is considered as a dense subset of $K(X)$.

Remarks 3.1. The following remarks are an immediate consequence of the definition of scattered spaces and the construction of compactification:

(1) Let $K(X)$ be a compactification of a topological space $X$. If $K(X)$ is scattered then $X$ is also a scattered space.

(2) Let $X$ be a scattered topological space and $A$ be a subset of $X$. Then the subspace $A$ is also scattered.

The following proposition is an immediate consequence of the Proposition 2.7

Proposition 3.2. Let $X$ be a topological space such that its compactification $K(X)$ is a $T_{0}$-space. Then $K(X)$ is scattered if and only if $X$ and $K(X)-X$ are scattered.

The most known compactification of a topological space is the one-point compactification (also called, the Alexandroff compactification). The one-point compactification is defined as follows: let $(X, T)$ be a non-compact topological space and $\infty \notin X$. Set $\widetilde{X}=X \cup\{\infty\}$. We equip $\widetilde{X}$ with the topology $\widetilde{T}$ whose elements are open subsets of $X$ and subsets $U$ of $\widetilde{X}$ such that $\widetilde{X}-U$ is a closed compact subset of $X$. The space with underlying set $\widetilde{X}$ and topology $\widetilde{T}$ is called the one-point compactification of $X$. Remark that if $X$ is a compact topological space then $\widetilde{X}$ is not a compactification of the space $X$ and it is called the Alexandroff extension of $X$ [7]. 
The following result gives a complete characterization of a space such that its one-point compactification is a scattered space. It is an immediate consequence of Proposition 3.2

Proposition 3.3. Let $X$ be a non-compact $T_{0}$-space. Then the one-point compactification $\widetilde{X}$ of $X$ is a scattered space if and only if $X$ is a scattered space.

Now let us recall, the Wallman compactification construction introduced in 1938 for $T_{1}$-spaces [11]: Let $(X, T)$ be a $T_{1}$-topological space.

A closed filter on $X$ is a collection $\mathcal{F}$ of non-empty closed sets with properties:

(1) $\mathcal{F}$ is closed under finite intersections,

(2) $P_{1} \in \mathcal{F}, P_{1} \subseteq P_{2}$ implies $P_{2} \in \mathcal{F}$.

Let $w X$ be the collection of maximal closed filters on $X$. Let $D$ be a closed set of $X$. Set $D^{*}=\{\mathcal{F} \in w X \mid D \in \mathcal{F}\}$. Then the collection $\mathcal{C}=\left\{D^{*} \mid D\right.$ is a closed set of $X\}$ is a base for the closed sets of a topology $T_{w}$ on $w X$.

For an open set $U$ of $X$, we define $U^{*}=\{\mathcal{F} \in w X \mid F \subseteq U$ for some $F$ in $\mathcal{F}\}$. It is easily seen that the collection $\left\{U^{*} \mid U\right.$ is an open set of $\left.X\right\}$ is a base for open sets of the topology $T_{w}$. The space with underlying set $w X$ and topology $T_{w}$ is called the Wallman compactification of $X$.

Recall that Kovar [9] has proved that the Wallman compactification remainder (that is, $w X-X$ ) is finite if and only if there exists $n \in \mathbb{N}$ such that the supremum of cardinalities of every collection of disjoint closed non-compact sets is lower than $n$.

Proposition 3.4. Let $X$ be a $T_{1}$-space such that its Wallman compactification remainder is finite. Then the Wallman compactification $w X$ is a scattered space if and only if $X$ is a scattered space.

P r o of. We have,

(1) Necessary condition: immediate.

(2) Sufficient condition: Since $w X-X$ is finite and $w X$ is a $T_{1}$-space, $w X-X$ is a discrete space. So that $w X-X$ is scattered. That $w X$ is a scattered space follows immediately from Proposition 2.7.

EXAMPLE 3.5. The assumption that the Wallman compactification remainder is finite in the Proposition 3.4 is essential. In fact: Let $\mathbb{N}$ be the discrete space of natural numbers. It is immediate that $\mathbb{N}$ is a scattered space and $w \mathbb{N}-\mathbb{N}$ is infinite. Now, since $\mathbb{N}$ is normal, $w \mathbb{N}$ coincides with the Stone Cech compactification $\beta \mathbb{N}$ of $\mathbb{N}$. Hence $w \mathbb{N}$ is not a scattered space.

The following lemma is an immediate consequence of the Wallman compactification construction. 
Lemma 3.6. Let $X$ be a $T_{1}$-space and $\mathcal{F} \in w X$. If $C$ is a closed set of $X$ such that $F \cap C \neq \emptyset$, for each $F \in \mathcal{F}$, then $C \in \mathcal{F}$.

Proposition 3.7. Let $X$ be a $T_{1}$-space such that its Wallman compactification is a scattered space. If $\mathcal{C}$ is a collection of disjoint closed non-compact sets of $X$, then there exists an open set $O$ of $X$ and $F \in \mathcal{C}$ such that:

(1) There exists a non-compact closed set $G$ of $X$ such that $G \subseteq F \cap O$.

(2) For each closed non-compact set $K \subseteq C$ with $C \in \mathcal{C}-\{F\}, K \nsubseteq O$.

P r o of. We have,

(1) Let $S=\{\mathcal{F} \in w X \mid \exists C \in \mathcal{C}, C \in \mathcal{F}\}$. Since $w X$ is scattered, there exists $\mathcal{F} \in S$ such that $\mathcal{F}$ is an isolated point in $S$. Hence there exists an open set $O$ of $X$ such that $O^{*} \cap S=\{\mathcal{F}\}$. Thus $\mathcal{F} \in O^{*}$; so that there exists $G \in \mathcal{F}$ such that $G \subseteq O$. Since $\mathcal{F} \in S$, let $F \in \mathcal{C}$ such that $F \in \mathcal{F}$. Then $G \subseteq F \cap O$.

(2) Let $C \in \mathcal{C}-\{F\}$ and $K$ be a closed non-compact set of $X$ such that $K \subseteq C$. Then there exists $\mathcal{K} \in S$ such that $K \in \mathcal{K}$. It is immediate that $\mathcal{K} \neq \mathcal{F}$, since elements of $C$ are disjoints; so that $\mathcal{K} \notin O^{*}$. Therefore $K \nsubseteq O$.

\section{Applications of scattered spaces to image classification}

The image classification problem, which involves assigning a label to each pixel of the observed image, is considered as a basic problem in mathematical imaging and is very useful in medical image processing. The image classification problem is to find a partition of the treated image into some regions such that each partition represents a class, that means a set of pixels with the same label. In this work, the label is chosen to be the grey level intensity. Moreover, assuming that both the number and parameters of these classes are known, we solve the problem of a supervised classification by grouping pixels having similar grey level intensity. The solution we obtain is a compound of some regions separated by boundaries. The classes are considered as phases separated by interfaces boundaries.

In this part, we are concerned with the problem of classifying a given original image $I_{0}$ according to $n$ predefined grey level intensities $C_{i}, 1 \leq i \leq n$. On the other hand, we mention that if $X$ is the domain on which $I_{0}$ is defined (an open bounded domain of $\mathbb{R}^{2}$ ), then $I_{0}$ is represented by an observed data function $I_{0}: X \rightarrow \mathbb{R}$ (the grey level intensity). The mathematical formulation of classifying $I_{0}$ using $n$ predefined classes $C_{i}, 1 \leq i \leq n$, such that the grey level intensity is chosen as a classifier can be written as: Find a family of open sets $\left\{O_{i} \mid i=1, \ldots, n\right\}$ verifying $O_{i} \cap_{i \neq j} O_{j}=\emptyset$ and $X=\cup_{i=1}^{n} O_{i} \cup \Gamma_{i}$, where

$$
\Gamma_{i}=\partial O_{i} \cap X
$$




\section{SCATTERED SPACES}

Let us note that $\Gamma_{i}$ is the boundary of the region $O_{i}$ except the common points with $\partial X$. Moreover, $\Gamma_{i}=\cup_{i \neq j} O_{i j}$, where $O_{i j}$ represents the interface between $O_{i}$ and $O_{j}$. We have,

$$
O_{i j}=O_{j i}=\Gamma_{i} \cap \Gamma_{j} \cap X, \quad \text { for all } i \neq j .
$$

To more clarify graphical representations of equations (4.1) and (4.2), we give an example of a partition of $X$ in Fig. 1

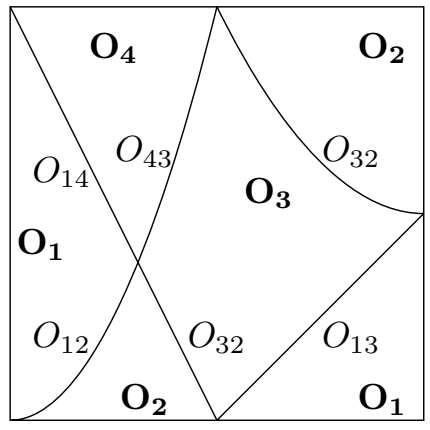

Figure 1. An example of a partition of $X$.

Finally, by the concept of a partition of $X$ given above, the image classification process consists in finding a partition $\left\{O_{i} \mid i=1, \ldots, n\right\}$ of $X$ such that $I_{0}$ is close to $c_{i}$ in $O_{i}$. The classified image $I$ will be defined by

$$
I(x)=c_{i} \quad \text { for all } \quad x \in O_{i} .
$$

A very simple way to realize such a classification is to apply the closest class algorithm that consists of creating clusters where elements within each cluster are similar, according to a predefined classifier. This classification process allows to select samples for each chosen class. Suppose for example that we aim to classify the given image $I_{0}$ according to the grey level intensity, by considering only two classes: $c_{1}=0$ (black) and $c_{2}=255$ (white). Then, the classified image is given by

$$
I(x)=c, \quad x \in X,
$$

such that

$$
c(x)= \begin{cases}c_{1}, & O_{1} \\ c_{2}, & X-\overline{O_{1}}\end{cases}
$$

To better illustrate this process, we give in Fig. 2(a) an initial scanner image of a human head. Fig. 2(b) shows the classified image using the closest class algorithm. 

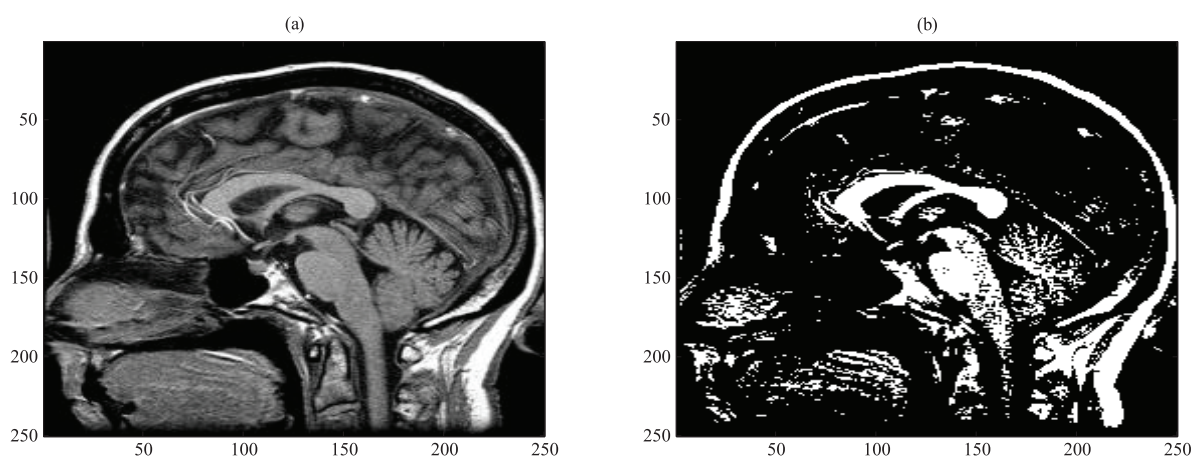

FiguRE 2. The initial image (a), the classified image using 2 classes $c_{1}=0$, $c_{2}=255$.

In order to generalize this process to $n$ classes with $n>2$, one can easily consider the equation (4.4), such that

$$
c(x)= \begin{cases}c_{1}, & O_{1}, \\ \vdots & \\ c_{n-1}, & O_{n-1}, \\ c_{n}, & X-\cup_{i=1}^{n-1} \overline{O_{i}},\end{cases}
$$

where $O_{i}$ represents the subset of pixels that should be reassigned to the class $c_{i}$. The unknowns are then the open subsets $O_{i}$ that constitute the partition of $X$. The obtained result shows a classified image $I$ that is close to the original one $I_{0}$.

From a numerical point of view, the image $I_{0}$ is defined by a finite element formulation. The domain is considered as a square and it is decomposed in a grid of pixels. The classification process is interpreted by an iterative process as follows:

- Input: initial image $I_{0}$.

- Compute isolated points of $I_{0} \Longrightarrow \mathcal{I}\left(I_{0}\right)$.

- For $i=1, \ldots, k$.

- Set $I_{i}=I_{i-1}-\mathcal{I}\left(I_{i-1}\right)$.

- Compute the isolated point of $I_{i} \Longrightarrow \mathcal{I}\left(I_{i}\right)$.

- Set $i=i+1$.

- Output: classified image $I=\cup_{i} I_{i}$.

One should note that if $X$ is scattered then this iterative process reaches the empty set. This final issue in this case gives an over-classification problem which clearly implies a non-regularized classification result. This represents a major drawback. In fact, an acceptable classification result is based on a regular and 


\section{SCATTERED SPACES}

homogeneous partition. A regular partition means that the union of all interfaces between two different subsets: $\Gamma_{i}=\cup_{i \neq j} O_{i j}$ is of minimal length. An homogeneous partition means that in each set $O_{i}$ the grey level intensity is close to $c_{i}$ in the $L^{2}$ norm sense. To overcome this inconvenience, regularization techniques should be used. To better visualize this increasing dispersion of classes, we give in Fig. 3 numerical tests showing the classification process by considering respectively the classes $\{0,50,100,150,200,255\}$ and then 256 classes such that all the grey level intensities from the black color $\left(c_{0}=0\right)$ to the white color $\left(c_{255}=1\right)$ are considered. We see that Fig. 3(b) for which all intensities are considered is similar to the original image $I_{0}$. This implies naturally an over-classification result and it is not accepted as a classification result. This over-classification is an immediate consequence of the fact that $X$ is a scattered space.
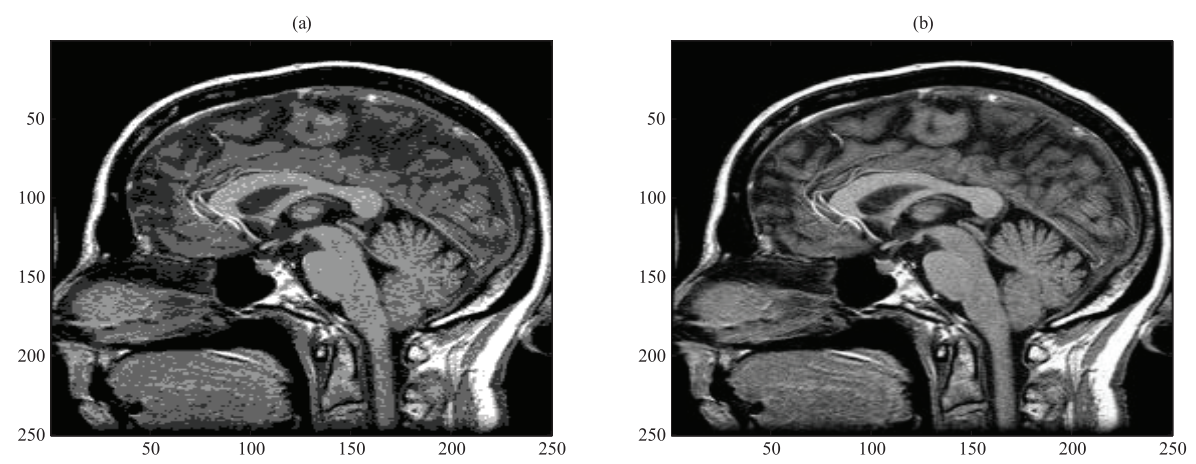

Figure 3. The classified image with the classes $\{0,50,100,150,200,255\}$ (a), the final result of classification using the concept of a scattered space $(b)$.

To avoid the result of an over-classification process obtained using the concept of scattered spaces, we can consider two different options:

- The first option is to regularize the classification process. The main goal of this regularization is to obtain smoother contours. Various methods are proposed in the literature in this context. The classical techniques and the most used ones are based on variational approaches. We refer the reader for example to [2], [12] for the use of such techniques. The principle is to minimize the following cost function

$$
g(O)=\sum_{i=1}^{n} \int_{O_{i}}\left(I_{0}(x)-c_{i}\right)^{2} d x,
$$


where $O=\left(O_{1}, O_{2}, \ldots, O_{n}\right)$ is a partition of $X$. This function $g$ measures the root mean square difference between the original image and the classified image. The principle of minimization consists in finding for each $x \in X$, the argument of the minimum $i_{x}=\operatorname{argmin}\left\{\left|I_{0}(x)-c_{i}\right|: i=1, \ldots, n\right\}$ and add $x$ to the subset $O_{i_{x}}$. In that way, each pixel of the original image is assigned in the classified image to its closest class. In order to obtain regularized results for the classification problem, other techniques based on the topological gradient have been used, [3].

- Since scattered spaces reaches automatically to an over-classification result, then we propose to study the case of more general spaces called the $S P$-scattered spaces, [6]. Let us first define such spaces.

Definition 4.1. Let $X$ be a topological space. An element $x$ of $X$ is said to be a $P$ point if the set of open neighborhoods of $x$ is closed under countable intersection.

Definition 4.2. Let $A$ be non-empty subset of $X$ and let $\mathcal{P}(A)$ be the set of $P$ points of $A . X$ is said to be $S P$-scattered if the interior of $\mathcal{P}(A)$ is non-empty for all $A \subseteq X$.

In fact, given a set of data points chosen according to their grey level intensity as a classifier, one should group clusters of points such that points in each cluster set are similar to each other. This similarity reaches to an automatic regularization of the classification result in comparison with that obtained by the scattered space concept. The numerical results of such regularization method are shown in Fig. 4. One should compare between the non-regularized image given by the Fig. 4 (a) and that shown by Fig. 4(b) which presents smoother contours.
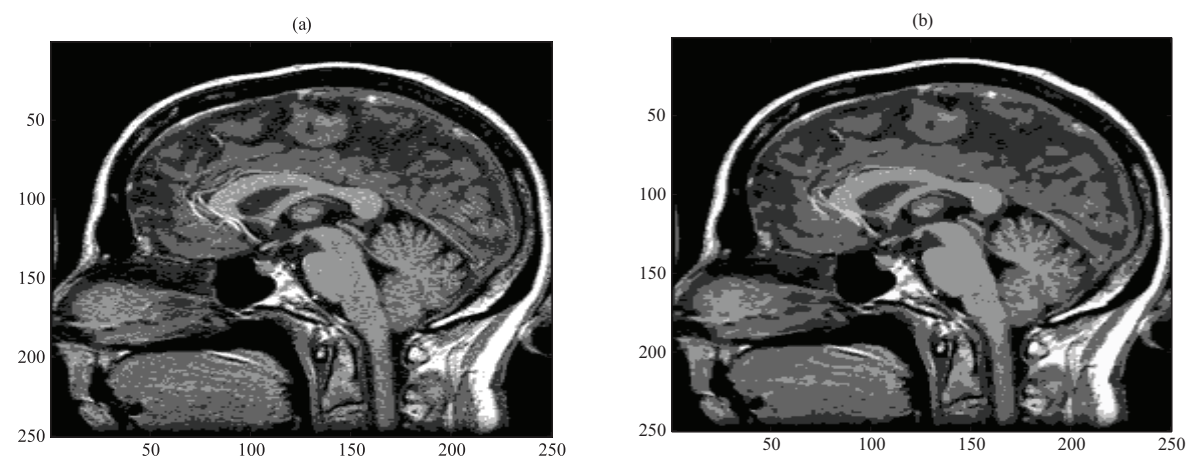

Figure 4. The non-regularized classified image (a), the final regularized result of classification (b).

As the $S P$-scattered spaces represent a remarkable advantage in classification and their use can be an alternative way for the regularization process, therefore 


\section{SCATTERED SPACES}

we propose in this last part to explore some properties of these spaces. More precisely, we give in the following proposition some properties of spaces such that their one-point compactification is the $S P$-scattered space.

Proposition 4.3. Let $X$ be a non-compact topological space and $\widetilde{X}$ its one-point compactification. Then the following statements are equivalent:

(1) $\mathcal{P}(A) \neq \emptyset$, for all $A \subseteq \tilde{X}$, with $A \neq \emptyset$.

(2) $X$ has the following properties:

- $\mathcal{P}(A) \neq \emptyset$, for all $A \subseteq X$, with $A \neq \emptyset$.

- The collection of compact closed sets of $X$ is closed under countable union.

Pr o of. We have,

$(1) \Longrightarrow(2)$ : That $\mathcal{P}(A) \neq \emptyset$, for all $A \subseteq X$, is straightforward. Now, let $\mathcal{C}$ be countable collection of closed sets $X$. Set $A=\widetilde{X}-\cup_{C \in \mathcal{C}} C$. Since $\mathcal{P}(A) \neq \emptyset$, for all $A \subseteq \tilde{X}$, there exits $a \in A$ such that $a$ is a $P$ point. The set $\mathcal{U}=$ $\{\widetilde{X}-C \mid \bar{C} \in \mathcal{C}\}$. Then $\mathcal{U}$ is a countable collection of open neighborhoods of $a$. Hence $A=\cap_{C \in \mathcal{C}}(\widetilde{X}-C)$ is an open set of $\widetilde{X}$ that contains $\infty$. Thus $\cup_{C \in \mathcal{C}} C$ is a compact closed set of $X$.

$(2) \Longrightarrow(1)$ : Let $A$ be a subset of $\widetilde{X}$. We consider two cases:

- Case 1: $A=\{\infty\}$. Since the collection of compact closed sets of $X$ is closed under countable union, $\infty$ is a $P$ point. So that $\mathcal{P}(\{\infty\}) \neq \emptyset$.

- Case 2: $A-\{\infty\} \neq \emptyset$. Hence $A-\{\infty\} \subseteq X$. Since $\mathcal{P}(A) \neq \emptyset$, for all $A \subseteq X, \mathcal{P}(A-\{\infty\}) \neq \emptyset$. Thus there exists $a \in A-\{\infty\}$ such that the set of open neighborhoods of $a$ in $X$ is closed under finite countable intersection. Let $\mathcal{U}$ be a countable collection of open neighborhoods of $a$ in $\tilde{X}$. We have two cases:

Case $i$ : There exists $U \in \mathcal{U}$ such that $\infty \notin U$. Then $\mathcal{U}_{1}=\{U-$ $\{\infty\} \mid U \in \mathcal{U}\}$ is a countable collection of neighborhoods of $a$ in $X$. Since $\mathcal{P}(A) \neq \emptyset$, for all $A \subseteq X, \cap_{U \in \mathcal{U}}(U-\{\infty\})$ is an open neighborhood of $a$. So that $\cap(U: U \in \mathcal{U})$ is an open neighborhood of $a$, since $\cap(U: U \in \mathcal{U})=\cap(U-\{\infty\}: U \in \mathcal{U})$.

Case $i i$ : For every $U \in \mathcal{U}, \infty \in U$. Hence $\mathcal{U}_{1}=\{U-\{\infty\} \mid U \in \mathcal{U}\}$ is a countable collection of neighborhoods of $a$ in $X$. Since $a$ is a $P$ point, $\cap_{U \in \mathcal{U}}(U-\{\infty\})$ is an open neighborhood of $a$. Since $X-\cap_{U \in \mathcal{U}}(U-\{\infty\})=\cup_{U \in \mathcal{U}}(X-(U-\{\infty\}))$ and $\{(X-(U-\{\infty\})) \mid U \in \mathcal{U}\}$ is countable collection of compact closed sets of $X, \cup_{U \in \mathcal{U}}(X-(U-\{\infty\}))$ is a compact closed set of $X$. Thus $\cap_{U \in \mathcal{U}} U=\widetilde{X}-\cup_{U \in \mathcal{U}}(X-(U-\{\infty\}))$ is an open neighborhood of $a$. 
The previous result incites us to ask the following questions.

Questions. Let $X$ be a space.

(1) When is the one-point compactification $\widetilde{X}$ of $X, S P$-scattered?

(2) When is the Wallman compactification $w X$ of $X, S P$-scattered?

\title{
REFERENCES
}

[1] AL HAJRI, M.-BELAID, K.-ECHI, O.: Stone spaces and compactifications, Pure Math. Sci. 2 (2013), 75-81.

[2] AUBERT, G.-KORNPROBST, P.: Mathematical problems in image processing, in: Appl. Math. Sci., Vol. 147, Springer-Verlag, New York, 2002.

[3] AUROUX, D.-JAAFAR BELAID, L.-MASMOUDI, M.: A topological asymptotic analysis for the regularized grey-level image classification problem, Math. Model. Numer. Anal. 41 (2007), 607-625.

[4] BELAID, K.-ECHI, O.-GARGOURI, R.: A-spectral space, Topology Appl. 138 (2004), 315-322.

[5] BELAID, K.: H spectral spaces, Topology Appl. 153 (2006), 3019-3023.

[6] HENRIKSEN, M.-RAPHAEL, R.-WOODS, R. G.: SP-scattered spaces; a new generalization of scattered spaces, Comment. Math. Univ. Carolin. 48 (2007), 487-505.

[7] KELleY, J.: General topology (2nd ed.), in: Grad. Texts in Math., Vol. 27, Springer-Verlag, New York, 1975.

[8] KHALIMSKY, E.-KOPPERMAN, R.-MEYER, P. R.: Computer graphics and connected topologies on nite ordered sets, Topology Appl. 36 (1990), 1-17.

[9] KOVAR, M. M.: Which topological spaces have a weak reflection in compact spaces? Comment. Math. Univ. Carolin. 36 (1995), 529-536.

[10] MROWKA, S.-RAJAGOPALAN, M.-SOUNDARARAJAN, T.: A characterization of compact scattered spaces through chain limits; (chain compact spaces). in: General Topology and its Applications-TOPO '72, 2nd Pittsburgh Internat. Conf., Pittsburgh, USA, 1972, Lecture Notes in Math., Vol. 378, Springer-Verlag, Berlin, 1974, pp. 288-297.

[11] WALlmAN, H.: Lattices and topological spaces, Ann. of Math. 2 (1938), 112-126.

[12] WEICKERT, J.: Efficient image segmentation using partial differential equations and morphology, Pattern Recognition 34 (2001), 1813-1824.

Received April 11, 2016

\author{
University of Dammam \\ College of Science \\ Department of Mathematics \\ P.O. Box 383 \\ Dammam 31113 \\ SAUDI ARABIA \\ E-mail: Mualhajri@uod.edu.sa \\ Kbelaid@uod.edu.sa \\ Ljaafar@uod.edu.sa
}

